\title{
Daño vascular asociado a uso de cocaína. Caso clínico
}

\author{
TERESA MASSARDO ${ }^{1}$, ÁNGELA PINO², ISABEL BERROCAL ${ }^{1}$, \\ GABRIEL CASTRO ${ }^{1}$, HERNÁN PRAT ${ }^{2}$, JAIME PEREIRA ${ }^{3}$

\section{Cocaine-induced vascular damage. Report of one case}

Cocaine abuse is associated with an increased risk of cardiac and cerebrovascular events, such as myocardial infarction, sudden cardiac death, and ischemic stroke. The underlying mechanisms leading to these complications are not fully understood although intravascular thrombus formation and accelerated atherosclerosis are prominent findings. We report a 39-year-old male addicted to cocaine, who presented with three consecutive ischemic events characterized by an acute myocardial infarction and two ischemic strokes complicated by cardiac failure and severe neurological sequelae. The pathophysiology of cocaine-induce vascular damage and the management of the ischemic complications are discussed.

(Rev Med Chile 2012; 140: 507-511).

Key words: Cocaine; Endothelium, vascular; Myocardial infarction.

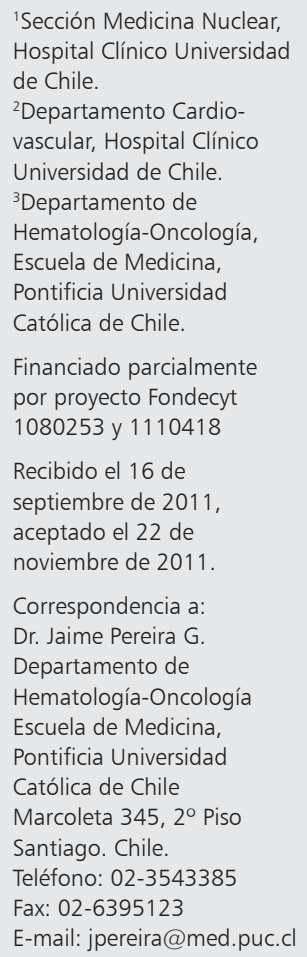

E 1 consumo de cocaína es un problema mayor a nivel mundial, dando origen a morbilidad, mortalidad y costos significativos a los sistemas de salud. En Chile, alrededor de 2\% de la población entre 12 y 64 años reconoce haber consumido cocaína el último año, fundamentalmente hombres, entre 19 y 34 años que pertenecen a estratos socioeconómicos medio y bajos ${ }^{1}$. Aparte de los efectos sociales y sicológicos, los usuarios crónicos de cocaína presentan complicaciones médicas graves, especialmente aquellas que afectan el aparato vascular, que pueden derivar en accidentes isquémicos serios que comprometen diversos territorios ${ }^{2}$. Datos publicados en Estados Unidos de Norteamérica y Australia muestran que el uso de cocaína representa la primera causa de muerte relacionada al uso de drogas ilícitas, afectando fundamentalmente a individuos entre $14 \mathrm{y}$ 34 años $^{2,3}$. El uso crónico de cocaína aumenta el riesgo de presentar infarto de miocardio, angina, muerte súbita y accidentes cerebrovasculares tanto isquémicos como hemorrágicos ${ }^{4,5}$.

\section{Caso clínico}

Hombre de 39 años, consumidor habitual por largo tiempo de cocaína, pasta base, marihuana y alcohol; además, tabaco y ocasionalmente benzodiacepinas; normopeso sin otros factores de riesgo cardiovascular convencionales. Posterior a ingesta de $1 \mathrm{~g}$ de cocaína y $3 \mathrm{~g}$ de pasta base presentó dolor retroesternal intenso. Hospitalizado por infarto agudo de miocardio anteroseptoapical con manejo médico, evolución tórpida y disfunción ventricular izquierda. Coronariografía efectuada a los quince días mostró oclusión ostial de arteria descendente anterior, sin circulación colateral (Figura 1a). Una resonancia magnética cardíaca confirmó infarto transmural anteroseptoapical sin evidencias de viabilidad, con signos de obstrucción microvascular, disfunción sistólica severa del ventrículo izquierdo (VI). Se inició tratamiento con heparina de bajo peso molecular (HBPM). No se logró manejo de dependencia por especialistas. Mes y medio más tarde presentó hemiparesia 


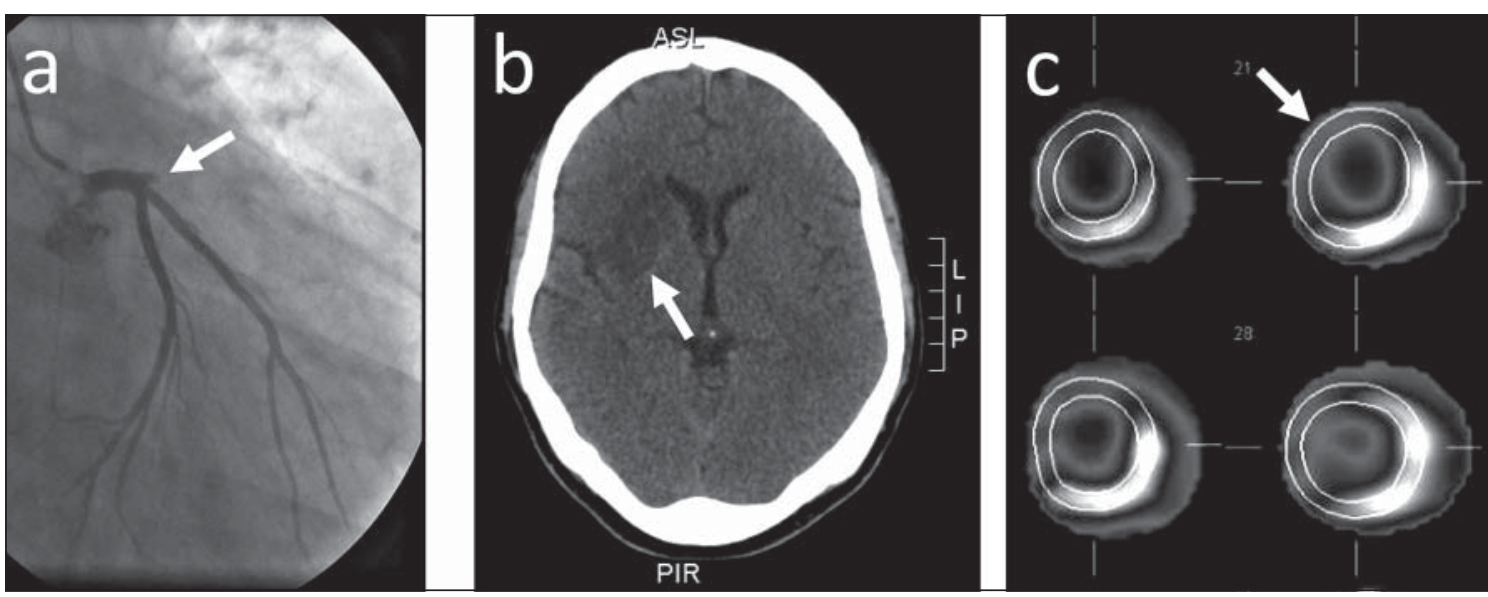

Figura 1. Estudios de imágenes que muestran compromiso de distintos territorios vasculares. Coronariografía contrastada (a) muestra arteria descendente anterior (DA) ocluida en su origen (flecha). Tomografía computada de cerebro (b), corte axial medio, con presencia de lesión parenquimatosa profunda frontolenticular insular derecha compatible con probable origen isquémico (flecha). SPECT con Sestamibi Tc ${ }^{99 m}$ (c) con cortes de eje con Dipiridamol e.v. (superior) y en reposo post nitritos (inferior) muesta gran defecto de perfusión de predominio fijo (flecha) anteroseptoapical en territorio DA, en relación a extenso infarto; también se aprecia dilatación de la cavidad izquierda en ambas fases.

facio braquio crural izquierda, catalogada como accidente isquémico transitorio y tratado ambulatoriamente. La sintomatología disminuyó parcialmente, asegurando abstinencia. Al mes siguiente, se hospitalizó por cuadro súbito de sudoración e hipotensión arterial, interpretado como síncope; se evidenció paresia facial central izquierda de tipo secuelar con buena sensibilidad y motilidad en las 4 extremidades. La tomografía computada cerebral mostró lesión parenquimatosa frontolenticular insular derecha de probable origen isquémico (Figura 1b). Se inició nuevamente terapia anticoagulante por alto riesgo embólico con HBPM. Debido a nueva akinesia inferior y adelgazamiento anteroseptoapical se efectuó SPECT de perfusión miocárdica con estrés farmacológico que demostró gran defecto fijo anteroseptoapical e inferoapical (aproximadamente 60\% del VI) con mínima isquemia periinfarto, sin viabilidad (Figura 1c). El ECG basal mostró retardo de conducción intraventricular. Posteriormente, se realizó prueba de esfuerzo Bruce submáxima bajo beta-bloqueo, no concluyente (7 METS), sin presentar angor. Evolucionó con leve dilatación de aurícula izquierda y marcada dilatación sistodiatólica del VI, akinesia apical e hipokinesia inferior, dilatación moderada de cavidades derechas, insuficiencias valvulares y cierto grado de hipertensión pulmonar. En análisis de asincronía hubo retardo significativo de conducción en segmentos medio-basal posteriores que planteó implante de desfibrilador automático, por importante remodelación del VI.

En comunidad terapéutica logró mejor manejo de dependencia. En control por insuficiencia cardíaca fue incluido en programa de trasplante cardíaco.

En resumen, paciente adulto joven, con dependencia principal a cocaína y pasta base, cuyo único factor de riesgo cardiovascular convencional era el hábito tabáquico. Presentó tres episodios consecutivos de eventos isquémicos coronarios y cerebrales en lapso corto con secuelas graves (insuficiencia cardíaca y motora facial); el manejo de su dependencia se logró tardíamente.

\section{Discusión}

El uso de cocaína es un reconocido factor de riesgo de daño vascular, que puede afectar diversos territorios aunque principalmente las complicaciones isquémicas se observan en sistemas cardio y cerebrovascular. Desde el punto de vista clínico, los usuarios crónicos de cocaína exhiben un mayor riesgo de angina de pecho, infarto agudo del miocardio, muerte súbita de origen cardíaco y accidentes vasculares cerebrales ${ }^{6,7}$.

La cocaína (benzoilmetilecgonina) es un alca- 
loide extraído de la hoja del arbusto eritroxilon coca. En nuestro medio, disponemos de clorhidrato de cocaína y "pasta base". El clorhidrato se utiliza en forma oral, intravenosa o intranasal. La "pasta base" se puede fumar. ${ }^{8}$ Cuando la droga es administrada sistémicamente, sus efectos están mediados por alteraciones a nivel de transmisión sináptica; la cocaína bloquea la reincorporación presináptica de norepinefrina y dopamina, con exceso de neurotransmisores en el sitio de los receptores en la neurona postganglionar, por lo que la cocaína actuaría como poderoso simpaticomimético.

\section{Fisiopatología}

Los mecanismos responsables del daño vascular asociado al uso de cocaína son múltiples y no están totalmente dilucidados. Principalmente debido a sus propiedades simpaticomiméticas, aumenta el consumo de oxígeno, produce taquicardia, eleva la presión arterial, aumenta la contractilidad miocárdica e induce vasoconstricción coronaria'. Sin embargo, los efectos vasomotores no explican por sí solos todas las complicaciones isquémicas observadas en estos pacientes, especialmente aquellas que ocurren en el largo plazo. Es importante tener en cuenta que el uso de cocaína puede inducir trombosis coronaria, observada hasta en $40 \%$ de los pacientes sometidos a angiografía por dolor torácico en relación a uso de cocaína ${ }^{10}$. Esta obstrucción trombótica que puede afectar distintos territorios vasculares está asociada al desarrollo de aterosclerosis prematura, la que se encontró en $76 \%$ de individuos con muerte súbita asociada al uso de esta droga ${ }^{7}$. Entre los elementos que determinan el inicio y progresión del daño vascular aterosclerótico la disfunción endotelial juega un papel fundamental ${ }^{11}$. Evidencia obtenida de estudios in vitro y en modelos animales demuestra que la cocaína induce disfunción de las células endoteliales ${ }^{12}$. En usuarios crónicos, hemos observado la existencia de disfunción endotelial importante demostrada por aumento significativo de esas células circulantes y niveles elevados de marcadores solubles de daño/activación de la célula endotelial ${ }^{13}$. Esta se mantiene en gran parte después de un mes de abstinencia estricta, lo que sugiere que el daño vascular inducido es de más largo plazo y no solamente asociado a los efectos agudos de la cocaína ${ }^{13}$. En este mismo sentido y en relación con las complicaciones trombóticas de la adicción, se ha demostrado activación de las plaquetas tanto en relación a uso agudo como crónico de cocaína ${ }^{14,15}$. En dependientes de cocaína observamos que después de consumo reciente existe activación de las plaquetas circulantes, demostrada por marcadores celulares y solubles, que regresan después de un mes de abstinencia ${ }^{16}$.

La información de la literatura y nuestras observaciones permiten sostener que en la patogenia de las complicaciones isquémicas inducidas por uso de cocaína, aparte de efectos agudos determinados por las propiedades simpaticomiméticas de la droga, existe daño endotelial que asociado a activación del sistema hemostático contribuyen no solamente a desencadenar eventos isquémicos sino que también al desarrollo y progresión del daño vascular.

\section{Diagnóstico y manejo de los eventos isquémicos}

El síntoma de consulta más frecuente en adictos a la cocaína es el dolor torácico, de los cuales hasta $6 \%$ puede presentar un infarto agudo de miocardio ${ }^{17}$. En la demostración de isquemia miocárdica en pacientes con dolor torácico asociado a cocaína (DTAC), se debe considerar en primer término, el electrocardiograma que es particularmente difícil de interpretar ya que hasta en $2 / 3$ de los casos de DTAC es inicialmente "anormal"18. Por otra parte, biomarcadores de necrosis como CK-total y CK-MB pueden estar elevados por rabdomiolisis, trauma o inyección intramuscular, por lo que en usuarios de cocaína con sospecha de infarto se recomienda uso de troponina $\mathrm{I}^{19}$.

De acuerdo a las guías clínicas de la Sociedad Americana de Cardiología (AHA) el manejo de los pacientes con dolor torácico e infarto de miocardio asociados a uso de cocaína es esencialmente similar a los no usuarios con algunas excepciones importantes, como es la contraindicación para uso de bloqueadores beta (Figura 2) ${ }^{20}$.

El diagnóstico y manejo clínico de los accidentes vasculares cerebrales relacionados a uso de cocaína no difiere en forma importante con relación a cuadros similares en pacientes no usuarios de drogas. Está demostrado que el uso de cocaína se asocia a un amplio espectro de cambios neuropatológicos, varios no bien caracterizados, que comprometen fundamentalmente el árbol vascular. Entre estos destaca el accidente vascular cerebral (AVC) considerado causa importante de morbilidad y mortalidad entre usuarios de cocaína ${ }^{21}$. 


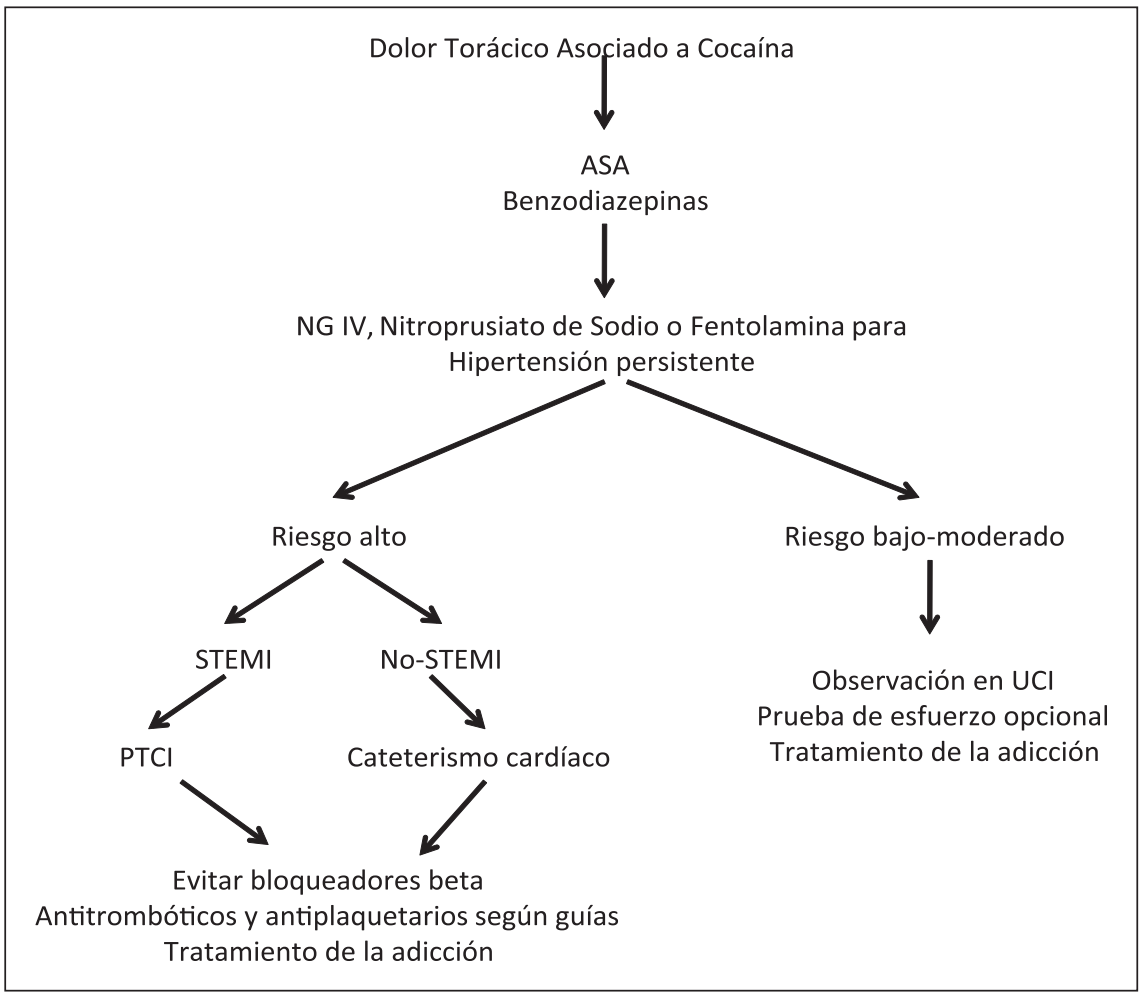

Figura 2. Recomendaciones diagnósticas y terapéuticas para el manejo del dolor torácico en usuarios de cocaína 20. ASA indica Aspirina ${ }^{\circledR}$; NG, nitroglicerina; STEMI, infarto de miocardio con elevación de ST; PTCl, angioplastia percutánea, $\mathrm{UCl}$, unidad de cuidado intensivo.
De hecho, la cocaína se considera la droga de uso ilícito más comúnmente asociada a enfermedad cerebrovascular en adultos jóvenes ${ }^{22}$; por ejemplo, en mujeres usuarias crónicas, el riesgo relativo de AVC puede alcanzar hasta 7 veces el de población pareada por edad ${ }^{21}$. La otra forma de evidenciar este daño cerebrovascular que parece ser mucho más frecuente que los accidentes vasculares, lo constituyen los defectos regionales de perfusión cerebral (DRPC) y la presencia de daño cognitivo de tipo ejecutivo ${ }^{23,24}$. Los AVC asociados al uso de cocaína se presentan en proporción relativamente similar entre isquémicos y hemorrágicos ${ }^{25}$. De los AVC de tipo isquémico, alrededor de $50 \%$ son secundarios a oclusión trombótica de arterias grandes y el resto a fenómenos embólicos y oclusión de vasos pequeños probablemente por vasculitis ${ }^{26}$.

En cuanto al manejo de los AVC asociados a uso de cocaína, no difiere mayormente del que se utiliza en no consumidores; aunque los datos de la literatura son limitados, no existe evidencia que contraindique el uso de trombolisis precoz en los pacientes que presentan AVC isquémicos en este contexto $^{27}$.
En conclusión, el uso de cocaína representa un importante factor de riesgo para complicaciones isquémicas cardíacas y cerebrales. Especialmente, se debe tener presente en adultos jóvenes, de preferencia de sexo masculino, sin factores de riesgo cardiovascular convencionales que se presentan con eventos vasculares isquémicos o alteraciones neurocognitivas, aunque inicialmente no reconozcan la adicción. Debido a que la persistencia del consumo mantiene y agrava el daño vascular, es fundamental incorporar al manejo de estos pacientes una terapia de rehabilitación precoz.

\section{Referencias}

1. CONACE. Octavo estudio de drogas en población general de Chile. 2008.

2. Lange RA, Hillis LD. Cardiovascular complications of cocaine use. N Engl J Med 2001; 345: 351-8.

3. Vasica G, Tennant CC. Cocaine use and cardiovascular complications. Med J Aust 2002; 177: 260-2.

4. Schwartz BG, Rezkalla S, Kloner RA. Cardiovascular effects of cocaine. Circulation 2010; 122: 2558-69. 
5. Treadwell SD, Robinson TG. Cocaine use and stroke. Postgrad Med J 2007; 83: 389-94.

6. Maraj S, Figueredo VM, Lynn Morris D. Cocaine and the heart. Clin Cardiol 2010; 33: 264-9.

7. Lucena J, Blanco M, Jurado C, Rico A, Salguero M, Vázquez R, et al. Cocaine-related sudden death: A prospective investigation in south-west Spain. Eur Heart J 2010; 31: 318-29.

8. Caterall WMK. Local anesthesics. In: Hardman JG GA, Limbird LE, ed. Goodman and Gilman's Pharmacologic basis of therapeutics. New York: McGraw-Hill; 1996: 331-8.

9. Kloner RA, Hale S, Alker K, Rezkalla S. The effects of acute and chronic cocaine use on the heart. Circulation 1992; 85: 407-19.

10. Dressler FA, Malekzadeh S, Roberts WC. Quantitative analysis of amounts of coronary arterial narrowing in cocaine addicts. Am J Cardiol 1990; 65: 303-8.

11. Le Brocq M, Leslie SJ, Milliken P, Megson IL. Endothelial dysfunction: From molecular mechanisms to measurement, clinical implications, and therapeutic opportunities. Antioxid Redox Signal 2008; 10: 163174.

12. Pradhan L, Mondal D, Chandra S, Ali M, Agrawal KC. Molecular analysis of cocaine-induced endothelial dysfunction: Role of endothelin-1 and nitric oxide. Cardiovasc Toxicol 2008; 8: 161-71.

13. Sáez CG, Olivares P, Pallavicini J, Panes O, Moreno $\mathrm{N}$, Massardo $\mathrm{T}$, et al. Increased number of circulating endothelial cells and plasma markers of endothelial damage in chronic cocaine users. Thromb Res 2011.

14. Heesch CM, Wilhelm CR, Ristich J, Adnane J, Bontempo FA, Wagner WR. Cocaine activates platelets and increases the formation of circulating platelet containing microaggregates in humans. Heart 2000; 83: 688-95.

15. Rinder HM, Ault KA, Jatlow PI, Kosten TR, Smith BR. Platelet alpha-granule release in cocaine users. Circulation 1994; 90: 1162-7.

16. Pereira J, Sáez CG, Pallavicini J, Panes O, Pereira-Flores $\mathrm{K}$, Cabreras MJ, et al. Platelet activation in chronic cocaine users: Effect of short term abstinence. Platelets. 2011.

17. Weber JE, Chudnofsky CR, Boczar M, Boyer EW,
Wilkerson MD, Hollander JE. Cocaine-associated chest pain: How common is myocardial infarction? Acad Emerg Med 2000; 7: 873-7.

18. Hollander JE, Lozano M, Fairweather P, Goldstein E, Gennis P, Brogan GX, et al. "Abnormal" electrocardiograms in patients with cocaine-associated chest pain are due to "normal" variants. J Emerg Med 1994; 12: 199-205.

19. Hollander JE, Shih RD, Hoffman RS, Harchelroad FP, Phillips S, Brent J, et al. Predictors of coronary artery disease in patients with cocaine-associated myocardial infarction. Cocaine-Associated Myocardial Infarction (CAMI) study group. Am J Med 1997; 102: 158-63.

20. McCord J, Jneid H, Hollander JE, de Lemos JA, Cercek B, Hsue P, et al. Management of cocaine-associated chest pain and myocardial infarction: A scientific statement from the American Heart Association Acute Cardiac Care Committee of the Council on Clinical Cardiology. Circulation 2008; 117: 1897-907.

21. Petitti DB, Sidney S, Quesenberry C, Bernstein A. Stroke and cocaine or amphetamine use. Epidemiology 1998; 9: 596-600.

22. Buttner A, Mall G, Penning R, Sachs H, Weis S. The neuropathology of cocaine abuse. Leg Med (Tokyo). 2003; 5 Suppl 1: S240-2.

23. Massardo T, Quintana JC, Jaimovich R, Pallavicini J, Servat M, Ibáñez C, et al. Basal brain perfusion defects and neurocognitive dysfunction in chronic cocaine users. Alasbimn J 2009; 46:45.

24. Massardo T, Pallavicini J, Quintana JC, Jaimovich R, Sáez CG, Olivares $\mathrm{P}$, et al. Brain perfusion defects are associated with endothelial dysfunction in cocaine users. J Nucl Med 2009; 50 (suppl 2): 1195.

25. Daras M, Tuchman AJ, Koppel BS, Samkoff LM, Weitzner I, Marc J. Neurovascular complications of cocaine. Acta Neurol Scand 1994; 90: 124-9.

26. Toossi S, Hess CP, Hills NK, Josephson SA. Neurovascular complications of cocaine use at a tertiary stroke center. J Stroke Cerebrovasc Dis 2010; 19: 273-8.

27. Martin-Schild S, Albright KC, Misra V, Philip M, Barreto $\mathrm{AD}$, Hallevi $\mathrm{H}$, et al. Intravenous tissue plasminogen activator in patients with cocaine-associated acute ischemic stroke. Stroke 2009; 40: 3635-7. 